\title{
A Highly Efficient Broadband Class-E Power Amplifier with Nonlinear Shunt Capacitance
}

\author{
Ninh Dang-Duy ${ }^{1} \cdot \mathrm{Nam} \mathrm{Ha} \mathrm{Van}^{1} \cdot$ Daesik Jeong $^{2} \cdot$ Dong Hwan Kim$^{2} \cdot$ Chulhun Seo $^{1, *}$
}

\begin{abstract}
A new approach to designing a broadband and highly efficient class-E power amplifier based on nonlinear shunt capacitance analysis is proposed. The nonlinear shunt capacitance method accurately extracts optimum class-E power amplifier parameters, including an external shunt capacitance and an output impedance, at different frequencies. The dependence of the former parameter on the frequency is considered to select an optimal value of external shunt capacitor. Then, upon determining the latter parameter, an output matching network is optimized to obtain the highest efficiency across the bandwidth of interest. An analytical approach is presented to design the broadband class-E power amplifier of a MOSFET transistor. The proposed method is experimentally verified by a $140-170 \mathrm{MHz}$ class-E power amplifier design with maximum added power efficiency of $82 \%$ and output power of $34 \mathrm{dBm}$.
\end{abstract}

Key Words: Broadband, Class-E Power Amplifier, MOSFET Transistor, Nonlinear Output Capacitance, PAE.

\section{INTRODUCTION}

A power amplifier $(\mathrm{PA})$ is always an important component in a transmitting chain of a wireless system, since its performance strongly influences the overall system features, including bandwidth, output power, efficiency, and linearity. In a modern PA design, wide bandwidth and high efficiency are two major requirements that increase the number of frequency bands and reduce thermal problems in the power devices and their sizes and auxiliary costs. A switch-mode class-E PA with shunt capacitance is attractive for these purposes, due to its high efficiency and simple topology [1, 2]. However, [1] and [2] only concentrate on simplifying the analysis of the class-E PA while neglecting the actual characteristic of the shunt capacitance. In fact, the operation of the linear shunt capacitance class-E PA in theory is completely different from its practical operation. Be- cause the parasitic drain-to-source capacitance of the switching device, which significantly contributes to the overall shunt capacitance in the operation, is nonlinear, it is necessary to take into account the nonlinear characteristics of this capacitance.

The theory of the class-E PA with a nonlinear capacitance was first published in Chudobiak's work [3] in a case of the grading coefficient $m=0.5$. Then several numerical analyses were performed with the grading coefficient $m \neq 0.5[4,5]$. In these articles, only a nonlinear shunt capacitance of the power transistor is considered. Suetsugu and Kazimierczuk [6] has proposed an analysis and a design procedure for the class-E PA with a shunt capacitance composed of both transistor nonlinear output capacitance and linear external capacitance. This theory has been verified in a design of a high-efficiency class-E PA for 13.56 $\mathrm{MHz}$ wireless power transmission application [7]. The study of frequency limitation based on the nonlinear shunt ca-

Manuscript received May 9, 2017 ; Revised September 16, 2017 ; Accepted September 23, 2017. (ID No. 20170509-020J)

${ }^{1}$ Deparment of Information Communication, Materials, and Chemistry Convergence Technology, Soongsil University, Seoul, Korea.

${ }^{2}$ Hanwha Systems, Seoul, Korea.

"Corresponding Author: Chulhun Seo (e-mail: chulhun@ssu.ac.kr)

This is an Open-Access article distributed under the terms of the Creative Commons Attribution Non-Commercial License (http://creativecommons.org/licenses/by-nc/4.0) which permits unrestricted non-commercial use, distribution, and reproduction in any medium, provided the original work is properly cited.

(c) Copyright The Korean Institute of Electromagnetic Engineering and Science. All Rights Reserved. 
pacitance theory has been presented in article [8], which showed the nonlinear dependence of frequency upon supply voltage, output power, and transistor characteristic. The performance as well as the topology of the class-E PA depends on the maximum frequency. An optimum performance is only addressed when the operating frequency does not exceed the maximum frequency. In the event that the operating frequency is lower than the maximum frequency, an added external shunt capacitor is necessary to obtain an optimal value of the shunt capacitor, as in the topology used in Suetsugu's work, whereas the topology used by Chudobiak [3] is optimum if the operation and maximum frequencies are equal.

The mentioned studies concentrated on designing a class-E PA at a single frequency using an output resonant circuit with a high unloaded quality factor to tune the operating frequency. In the present work, a practical approach to designing a broadband high-efficiency class-E PA based on the nonlinear shunt capacitance theory is proposed. Applying nonlinear design equations from previous works $[6,8]$, the dependence of class-E PA operation on the frequency is focused on extracting the optimal values of the circuit parameters, including external shunt capacitance and output impedance. The topology of a low-pass matching network is utilized to overcome the restriction of the output resonant circuit for the broadband design. A prototype of a broadband class-E PA is implemented to validate the theoretical analysis.

\section{ANALYZING AND DESIGNING BROADBAND CLASS-E PA}

A conventional circuit of the MOSFET class-E PA with shunt capacitance is illustrated in Fig. 1(a). Without an input driving signal, a DC-voltage $V_{G S}$ is applied to the gate of the transistor to turn the transistor off. A DC-supply voltage $V_{D D}$ connects to the drain of the transistor through the choke coil $L_{R F C}$ with the only purpose of supplying DC power to the transistor. A shunt capacitor $C_{P}$, in parallel with the transistor, keeps a sinusoidal current waveform to alternate flow between the transistor and the shunt capacitor $C_{P}$. In common, $C_{P}$, consisting of a nonlinear shunt capacitance of a transistor $C_{d s}$ and a linear external capacitance $C_{e}$, is described as follows:

$$
C_{P}=C_{d s}+C_{e}=\frac{C_{j 0}}{\sqrt{1+\frac{v_{S}}{V_{b i}}}}+C_{e}
$$

where $C_{j 0}$ is the shunt capacitance at the drain-to-source voltage $v_{s}=0 \mathrm{~V}$, and $V_{b i}$ is the built-in potential of the MOSFET body diode. A series-resonant $C_{0} L_{0}$ circuit tunes to the fundamental

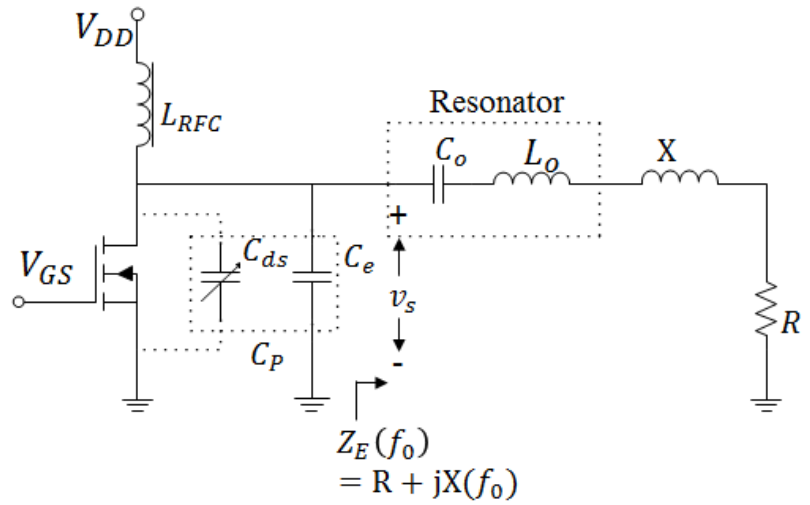

(a)

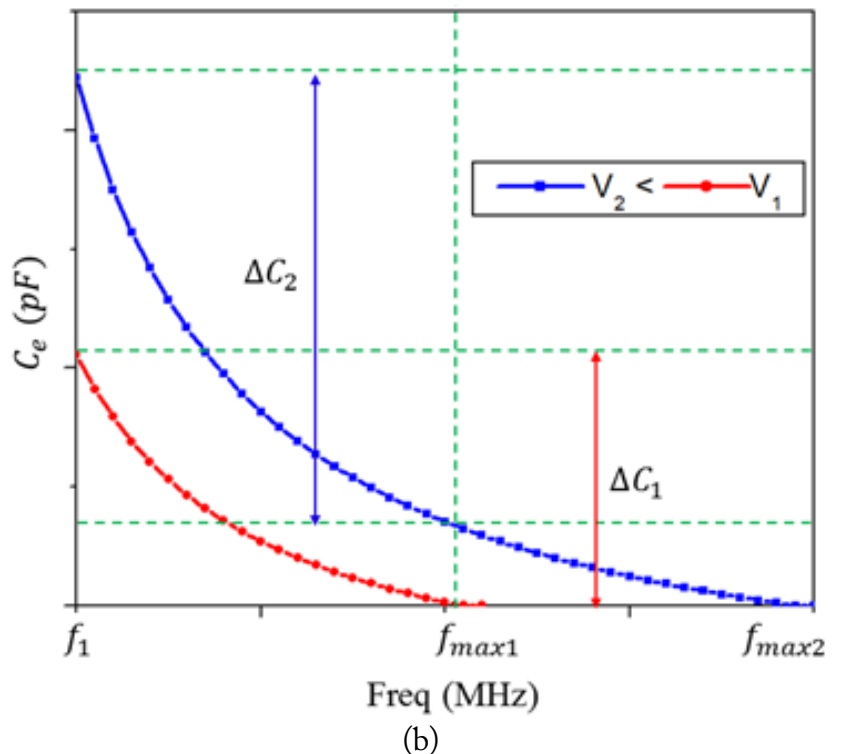

Fig. 1. The MOSFET class-E PA with shunt capacitance. (a) Conventional topology. (b) $C_{e}$ versus $f_{0}$ for selected $V_{D D}$.

frequency with a high unloaded quality factor $Q$ to shape the output current to be sinusoidal at the fundamental frequency. At the fundamental frequency $f_{0}$, a load $Z_{E}$ is inductive, as shown in Fig. 1(a). An optimal value of $Z_{E}$ guarantees that there is no overlap between the transient drain current and the voltage.

In previous works $[6,8]$, the design equations expressed the relationship among the operating frequency $f_{0}$, the supply voltage $V_{D D}$, the output power $P_{o}$, the external linear capacitance $C_{c}$, and the characteristic parameters of MOSFET, $C_{j 0}, V_{b i}$. The numerical solutions, a set of tables and figures, illustrate that relationship. For the purpose of broadband design, this work gives a practical scenario in which a specification of class$\mathrm{E} P A$ is defined as containing the operating frequencies, the output power, and the model of the MOFET transistor. Based on this specification, the analysis concentrates on the dependence of the circuit parameters on the operating frequency and the DC-supply voltage. For selected values of output power $P_{o}$ 
and MOSFET transistor parameters $C_{\mathfrak{j} 0}, V_{b i}$, and for swept values of operating frequency $f_{0}$ and $\mathrm{DC}$-supply voltage $V_{D D}$, this work re-solves the nonlinear design equation to extract the values of external linear capacitance $C_{e}$ and fundamental load $Z_{E}$. Then the optimal values of these parameters are chosen to obtain the highest efficiency of class-E PA.

\section{Selecting $V_{D D}$ to Obtain the Actual Value of $C_{e}$}

Fig. 1(b) plots the linear external $C_{e}$ as a function of the operating frequency $f_{0}$ and the DC-supply voltage $V_{D D} . C_{e}=0$ at the maximum operating frequency $f_{\max }$. To obtain high efficiency for a broadband design, the upper frequency $f_{U}$ of the bandwidth should be less than or equal to $f_{\text {max }}$. At the same conditions of $P_{o}$ and transistor, the increased $V_{D D}$ results in the decrement of $f_{\max }$. The theory of the maximum frequency of class-E PA with the nonlinear shunt capacitance expressing the relation between the maximum frequency and the supply voltage was presented in detail in another work [8].

In the broadband design, the optimum external capacitance varies with the frequency. Unfortunately, the actual value of the external capacitor is a constant; therefore, the optimum state is unattainable at every frequency. Simply, the actual $C_{e}$ is chosen as the average value of the optimum $C_{e}$. To approximate the idealized class-E PA mode, the deviations of the actual $C_{e}$ from the design values, depending on the variation of the optimum $C_{e}$ with the $f_{0}$, should be minimized. The small variation can result in the decreased level of the deviation. As shown in Fig. 1(b), at a given specification, the variation at a low band is much greater than that at a high band, whereas, at a given frequency band, from $f_{1}$ to $f_{\max }$, the variation will be smaller in the case of a high voltage, $\Delta C_{2}>\Delta C_{1}$. Hence, combined with the maximum frequency condition as mentioned above, $V_{1}$ corresponding to $f_{\max 1}$ is the optimal supply voltage with a bandwidth of $f_{1}$ to $f_{\max }$.

\section{Output Matching Network}

For most practical applications, it is necessary to match the required class-E PA optimum load impedance $Z_{E}$ with a standard load resistance $R_{L}$. In broadband design, the conventional topology of a class-E power amplifier is not proper for obtaining broadband design, due to the limitation of the series resonator, which requires a high $\mathrm{Q}$ factor to perfectly prevent harmonic frequency.

Low-pass matching networks (LMNs), one of the common ways of designing broadband class-E PAs, have shown high performance in published papers $[1,2]$. The LMN has two functions of low-pass filter, as a function of a resonant circuit and a matching network. Since a higher order of low-pass filter leads to wider bandwidth and steeper stopband attenuation, by increasing the value of the order, the LMN can overcome the bandwidth limitation of the resonator. However, the tradeoff between performance and the size of the low-pass filter, which depends on the requirements of the specifications, should be considered. The LMN also makes the circuit simpler by substituting both for the resonator and the output matching network in the conventional topology of a class-E PA.

The efficiency of an LMN depends on the accuracy in extracting $Z_{E}$. In the conventional method $[1,9,10], Z_{E}$ is expressed as follows:

$$
Z_{E}=\frac{0.88 V_{D D}^{2}}{P_{o}} e^{j 49^{\circ}}
$$

This expression was set up with the assumption that the linear shunt capacitance is inaccurate for application to MOSFET devices. As shown in Fig. 1(a), the resonant circuit, representing a short and an open circuit at the fundamental and harmonic frequencies, respectively, has zero reactance at the fundamental frequency; therefore, $Z_{E}$ is expressed by a fundamental inductive load that consists of $R$ and $X$ in series:

$$
\begin{gathered}
R=\frac{8 V_{D D}^{2}}{\left(\pi^{2}+4\right) P_{o}} \\
X=R \tan \left(\phi_{1}-0.567\right)
\end{gathered}
$$

where $\phi_{1}$ is the phase of $v_{s}$. Because the reactance of the resonator is zero at the operating frequency, the relation between $\phi_{1}$ and $v_{s}$ is described by:

$$
\frac{1}{\pi} \int_{0}^{2 \pi} v_{s}(\theta) \cos \left(\theta+\phi_{1}\right) d \theta=0
$$

Hence, the optimal load impedance is

$$
Z_{E}=\frac{8 V_{D D}^{2}}{\left(\pi^{2}+4\right) P_{o}}+j \frac{8 V_{D D}^{2}}{\left(\pi^{2}+4\right) P_{o}} \tan \left(\phi_{1}-0.567\right)
$$

While $R$ is only a function of $V_{D D}$ and $P_{o}, X$ depends on $V_{D D}$, $P_{o}, f_{0}$, and the characteristic of the transistor, different from the conventional formula. Similar to the method of extraction of $C_{e}$, the MATLAB program is composed to solve the nonlinear equation of $X$. Due to the dependence of the output impedance on the frequency, the $\mathrm{LMN}$ is required to match the various $Z_{E}$ to the standard load. The simple method to design the various output impedance LMNs is to use the Impedance Matching Utility Tool of the Advanced Design System (ADS).

\section{Design Procedure}

From the above analysis, the design steps of the broadband class-E PA are summarized as follows:

1) Based on specifications of the operating frequencies and expected output power, select the type of transistor.

2) Using the MATLAB program to solve the nonlinear de- 
sign equations, plot $C_{e}$ as a function of $f_{0}$ and $V_{D D}$, as shown in Fig. 1(b).

3) Select $V_{D D}$ to obtain the actual value of $C_{e}$. The rule of $V_{D D}$ selection is as follows: supposing the bandwidth of design class-E PA from $f_{1}$ to $f_{2}$, the optimal $V_{D D}$ has a value whose corresponding maximum frequency $f_{\max }$ is equal to $f_{2}$. Then, the actual value of $C_{e}$ is chosen as an average value of the optimal $C_{e}$ at every frequency of bandwidth.

4) Use Eqs. (5)-(6) to extract $Z_{E}$. Select the order of LMN based on the specification of bandwidth. Synthesize LMN by using the Impedance Matching Utility Tool of ADS.

\section{SIMULATION AND EXPERIMENT RESULTS}

To verify the above analysis, a prototype of class-E PA circuit was designed for public and homelands security application with $P_{\circ}$ of $34 \mathrm{dBm}$ over a bandwidth of $140-170 \mathrm{MHz}$. The MOSFET used in this design was MRF282SR1, with $C_{j 0}$ described by the following:

$$
C_{j 0}=\left(C_{o s s}-C_{r s s}\right) \sqrt{1+\frac{V_{s p e c}}{V_{b i}}}
$$

where $C_{\text {oss }}$ and $C_{r s s}$, respectively, are the output and reverse transfer capacitances of the transistor, (often provided in catalogs by power MOSFET manufacturers), and $V_{\text {spec }}$ is a special value of supply voltage used to measure the values of $C_{\text {oss }}$ and $C_{r s s}$. Using MATLAB, the $C_{e}$ as a function of $f_{0}$ for selected values of $V_{D D}$ was plotted in Fig. 2. From Fig. 2, the actual $V_{D D}$ and $C_{e}$ were 8 $\mathrm{V}$ and $1.2 \mathrm{pF}$, respectively. Eqs. (5)-(6) were solved using MATLAB to determine the output impedance at various frequencies. Fig. 3 plots the output impedance versus the operating frequency. By setting the output impedances at corresponding frequencies, the ADS Impedance Matching Tool synthesized

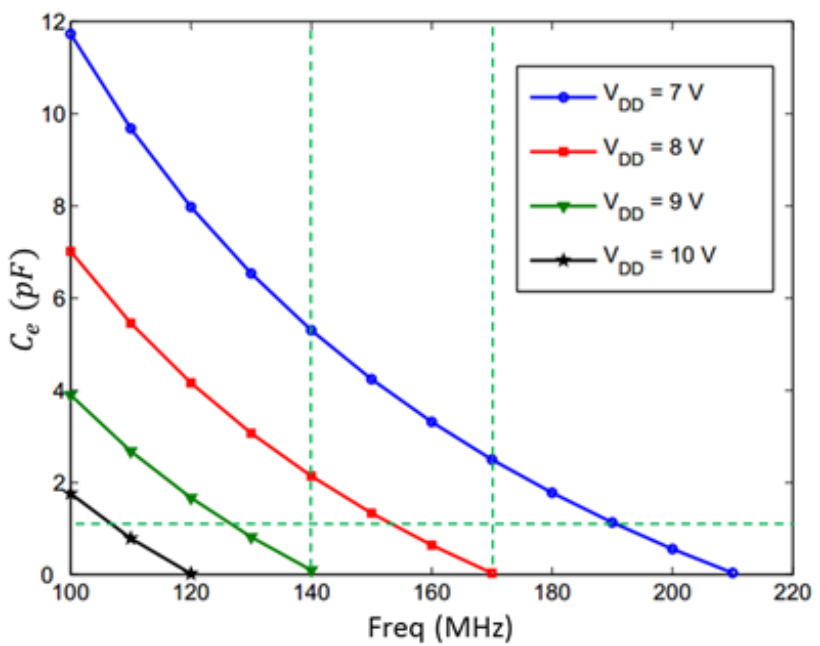

Fig. 2. Determining the optimal $V_{D D}$ and $C_{e}$.

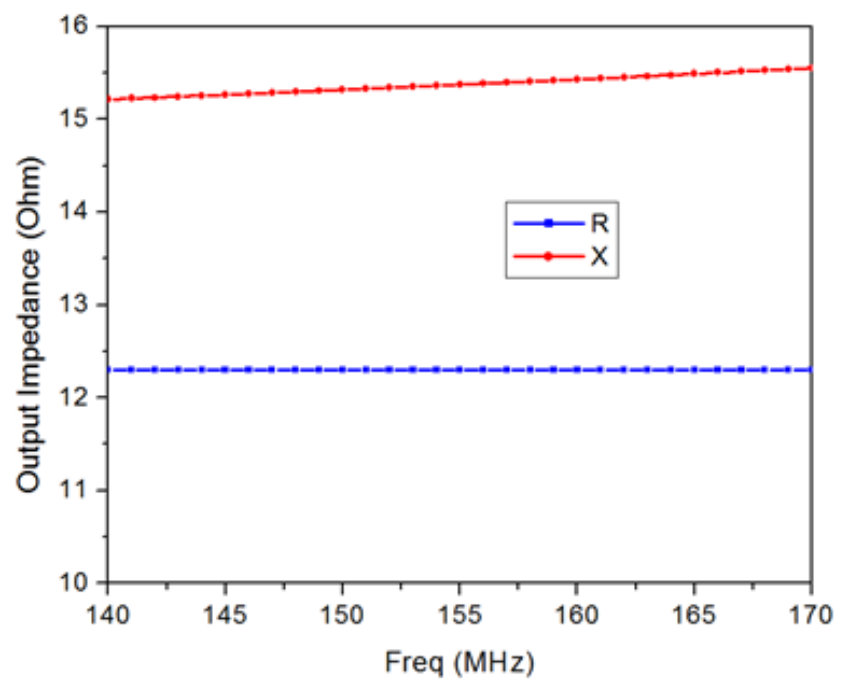

Fig. 3. Fundamental output impedance.

the matching network. A three-stage LMN with a bandwidth of $20 \%$ was extracted, illustrated in Fig. 4 . The simulated drain minimized power dissipation on the transistor. A voltage and current waveforms depicted in Fig. 5 under the optimum operation slightly overlap, resulting in a prototype of class-E PA circuit was realized on a Taconic TLC PCB material with a thickness of $0.787 \mathrm{~mm}$ and a dielectric constant of 3.2. A photograph of the prototype is shown in Fig. 6. The output power was measured using an Agilent 85665EC spectrum analyzer with a

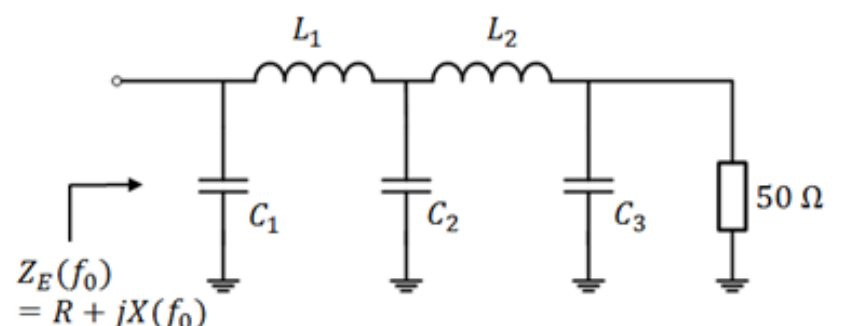

(a)

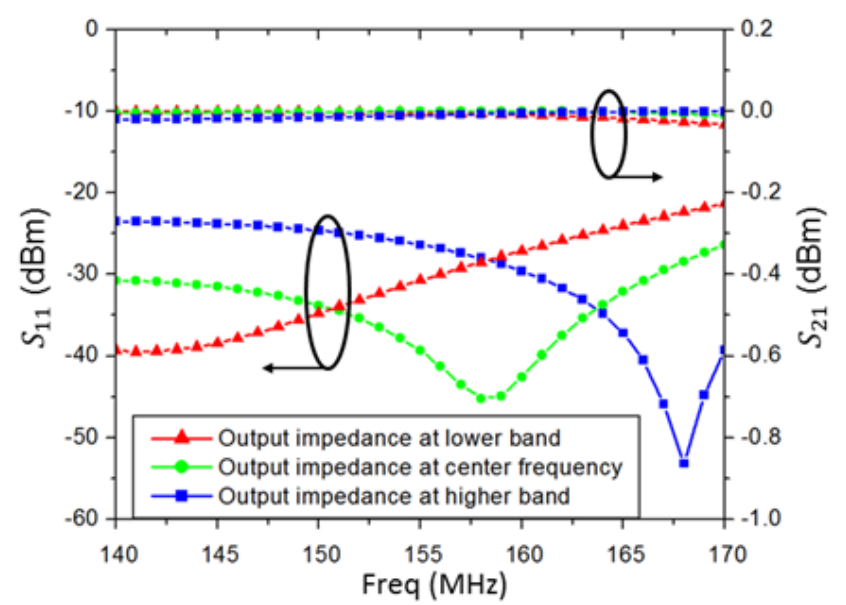

(b)

Fig. 4. The low-pass matching network. (a) Schematic. (b) $S$-paameters at different output impedance. 


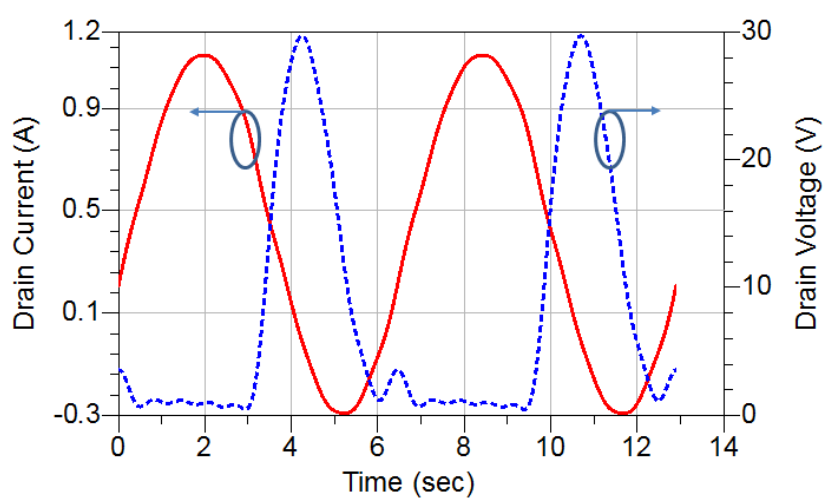

Fig. 5. Drain current and voltage simulation waveform of class-E PA circuit.

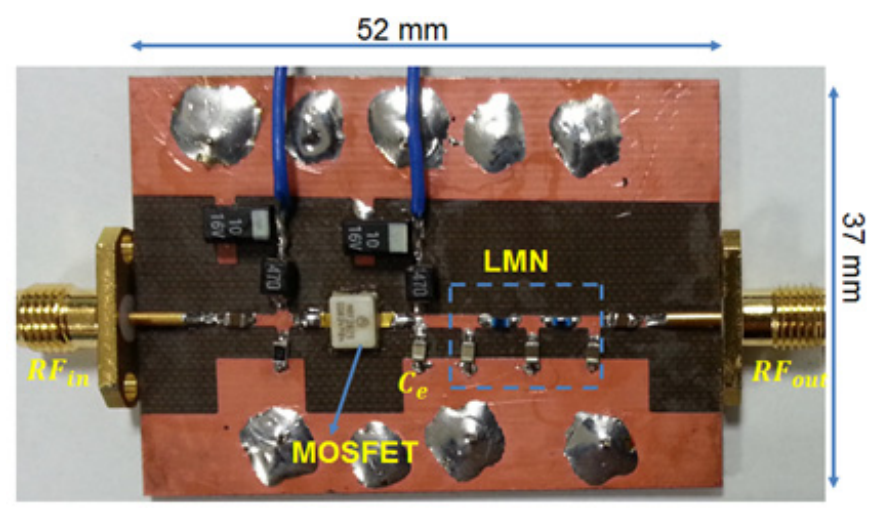

Fig. 6. Photograph of fabricated MOSFET class-E PA.

maximum measurement output of $30 \mathrm{dBm}$. An attenuator of $39.67 \mathrm{~dB}$ was added at the end of the circuit. The PAE and output power versus the frequency and input power were plotted in Fig. 7. The lumped components are available only in discrete values provided by the manufacturers, which is the main reason for the differences between the simulated and measured results. A summary of the experimental results compared with other articles on high efficiency proving this method's advantages is shown in Table 1.

\section{CONCLUSION}

This work presented a practical method to implement a high-

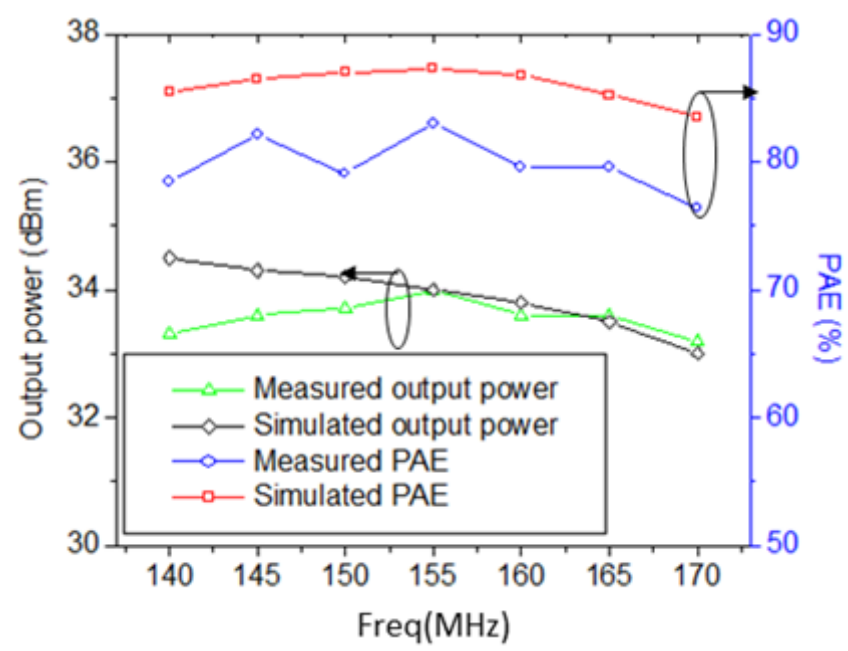

(a)

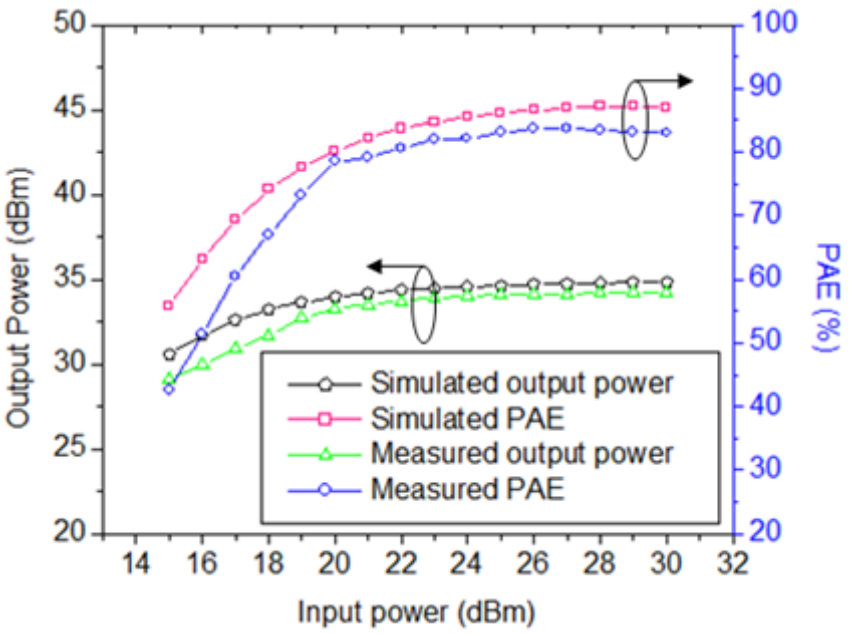

(b)

Fig. 7. Simulated and measured results. (a) $P_{o}$ and PAE versus $f_{0}$. (b) $P_{o}$ and PAE versus $P_{i n}$

efficiency broadband class-E PA using a MOSFET transistor with nonlinear output capacitance. The extraction of the shunt capacitance and the fundamental output impedance via numerical analysis was the principal step in this article. The peak PAE of $82 \%$ and the average PAE of $80 \%$ with the output power level of $34 \mathrm{dBm}$ at frequencies from $140 \mathrm{MHz}$ to $170 \mathrm{MHz}$ were experimental results that validated the accuracy of this method. Two of the most outstanding advantages of the class-E

Table 1. Comparative results

\begin{tabular}{|c|c|c|c|c|c|c|c|}
\hline Ref. & Year & $\begin{array}{c}\text { Frequency } \\
(\mathrm{MHz})\end{array}$ & $\begin{array}{c}\mathrm{BW} \\
(\mathrm{MHz})\end{array}$ & $\begin{array}{c}P_{\text {out }} \\
(\mathrm{dBm})\end{array}$ & $\begin{array}{c}\text { Efficiency } \\
(\%)\end{array}$ & $\begin{array}{c}\text { PAE } \\
(\%)\end{array}$ & Transistor \\
\hline [11] & 1987 & $130-180$ & 50 & 37.8 & 60.0 & - & MOSFET \\
\hline$[12]$ & 2008 & $136-174$ & 38 & 39.0 & 74.0 & - & MOSFET \\
\hline [13] & 2008 & $135-175$ & 40 & 38.1 & 70.0 & - & MOSFET \\
\hline [14] & 2010 & $140-170$ & 30 & 33.9 & - & 72.5 & GaN HEMT \\
\hline$[15]$ & 2010 & $134-174$ & 40 & 37.4 & 71.0 & - & MOSFET \\
\hline [16] & 2016 & 130-180 & 50 & 34.3 & 90.5 & 84.4 & MOSFET \\
\hline This work & & $140-170$ & 30 & 34.0 & 91.2 & 82.0 & MOSFET \\
\hline
\end{tabular}


power amplifier with shunt capacitance as high efficiency and simple topology were shown in this work.

This work was supported by a grant-in-aid of Hanwha Systems.

\section{REFERENCES}

[1] K. Chen and D. Peroulis, "Design of highly efficient broadband class-E power amplifier using synthesized low-pass matching networks," IEEE Transactions on Microwave Theory and Techniques, vol. 59, no. 5, pp. 3162-3173, 2011.

[2] O. Kizilbey, "Design of class-E GaN HEMT power amplifier using elliptic low pass matching network with $86 \%$ efficiency," IEICE Electronics Express, vol. 10, no. 2, article no. 20120960, 2013.

[3] M. J. Chudobiak, "The use of parasitic nonlinear capacitors in class E amplifier," IEEE Transactions on Circuits and Systems I: Fundamental Theory and Applications, vol. 41, no. 12, pp. 941-944, 1994.

[4] M. K. Kazimierczuk and D. Czarkowski, "Zero-voltageswitching resonant inverter," in Resonant Power Converter. New York, NY: McGraw-Hill, 1995.

[5] R. M. Kielkowski, SPICE: Practical Device Modeling. New York, NY: Wiley, 1995.

[6] T. Suetsugu and M. K. Kazimierczuk, "Analysis and design of class $\mathrm{E}$ amplifier with shunt capacitance composed of nonlinear and linear capacitances," IEEE Transactions on Circuits and Systems I: Regular Papers, vol. 51, no. 7, pp. 12611268, 2004.

[7] H. V. Nam and C. Seo, "Design of high PAE class-E power amplifier for wireless power transmission," IEICE Electronics Express, vol. 11, no. 17, article no. 20140682, 2014.

[8] H. V. Nam, D. D. Ninh, H. Kim, and C. Seo, "Frequency limitation of an optimum performance class-E power amplifier," IEICE Electronics Express, vol. 11, no. 7, article no. 20160108, 2016.

[9] T. B. Mader, E. W. Bryerton, M. Markovic, M. Forman, and Z. Popovic, "Switched-mode high-efficiency micro wave power amplifiers in a free-space power combiner broadband class-E PA using a MOSFET transistor with nonlinear output capacitance. The extraction of the shunt array," IEEE Transactions on Microwave Theory and Techniques, vol. 46, no. 10, pp. 1391-1398, 1998.

[10] E. Cipriani, P. Colantonio, F. Giannini, and R. Giofre, "Optimization of class E power amplifier design above theoretical maximum frequency," in Proceedings of 38th European Microwave Conference (EuMC), Amsterdam, the Netherlands, 2008, pp. 1541-1544.

[11] J. K. A. Everard and A. J. King, "Broadband power efficient class $\mathrm{E}$ amplifiers with a non-linear CAD model of the active MOS device," Journal of the Institution of Electronic and Radio Engineers, vol. 57, no. 2, pp. 52-58, 1987.

[12] N. Kumar, C. Prackash, A. Grebennikov, and A. Mediano, "High-efficiency broadband parallel-circuit class E RF power amplifier with reactance-compensation technique," IEEE Transactions on Microwave Theory and Techniques, vol. 56, no. 3, pp. 604-612, 2008.

[13] K. Narendra, A. Al Tanany, L. Anand, G. Boeck, C. Prakash, A. Grebennikov, and A. Mediano "High efficiency broadband class E RF power amplifier with high harmonics suppression for practical two-way radio applications," in Proceedings of 2008 German Microwave Conference (GeMIC), Harburg, Germany, 2008, pp. 1-4.

[14] E. Khansalee, N. Puangngernmak, and S. Chalermwisutkul, "A high efficiency VHF GaN HEMT class E power amplifier for public and homeland security applications," in Proceedings of 2010 Asia-Pacific Microwave Conference, Yokohama, Japan, 2010, pp. 437-440.

[15] K. Narendra, A. Mediano, L. Anand, and C. Prakash, "Second harmonic reduction in broadband HF/VF/UHF class E RF power amplifiers," in Proceedings of IEEE MTT$S$ International Microwave Symposium Digest, Anaheim, CA, 2010, pp. 328-331.

[16] D. D. Ninh, H. V. Nam, H. Kim, and C. Seo, "Design of a highly efficient broadband class-E power amplifier with a low Q series resonance," Journal of Electromagnetic Engineering and Science, vol. 16, no. 3, pp. 143-149, 2016. 


\section{Ninh Dang-Duy}

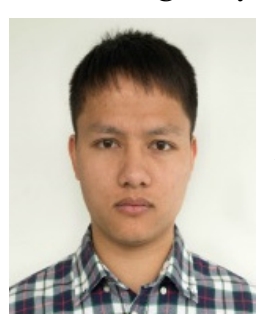

received his B.S. degree from the School of Electronics and Telecommunications at Hanoi University of Science and Technology, Hanoi, Vietnam in 2013. He is currently pursuing an Integrated Master's Ph.D. degree in the Department of Information Communication, Materials, and Chemistry Convergence Technology at Soongsil University, Seoul, Korea. His current research interests are power amplifiers, metamaterials, and wireless power transfer.

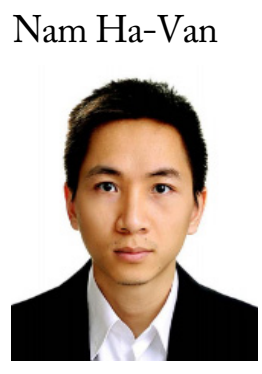

received his B.S. degree from the School of Electronics and Telecommunications at Hanoi University of Science and Technology, Hanoi, Vietnam in 2012. $\mathrm{He}$ is currently pursuing an Integrated Master's Ph.D. degree in the Department of Information Communication, Materials, and Chemistry Convergence Technology at Soongsil University, Seoul, Korea. His current research interests are wireless power transfer, wireless power transfer for biomedical implants, metamaterials, power amplifiers, dual-band RFID antenna, and phased array antennas.

\section{Dong Hwan Kim}

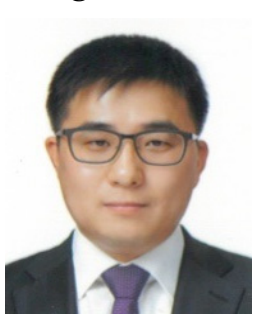

received the B.S. degree in electronic engineering from Inje University, Gimhae, Korea, in 2005, and the M.S. degree in electronic engineering from $\mathrm{Ky}^{-}$ ungpook National University, Daegu, Korea, in 2007. $\mathrm{He}$ is currently working at Hanwha Systems. His research interests include active phased array antenna design and multi-function radar system.

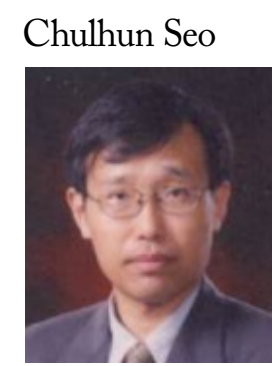

(M'97-SM'14) received B.S., M.S., and Ph.D. degrees from Seoul National University, Seoul, Korea, in 1983, 1985, and 1993, respectively. From 1993 to 1995, he was with the Massachusetts Institute of Technology (MIT), Cambridge, MA, USA, as a Technical Staff Member. From 1993 to 1997, he was with Soongsil University, Seoul, Korea, as an assistant professor. From 1999 to 2001, he was with MIT as a visiting professor. From 1997 to 2004, he was with Soongsil University as an associate professor. Since 2004, he has been a professor of electronic engineering with Soongsil University. He was IEEE MTT Korea Chapter chairman from 2011 to 2014. He is the president of the Korean Institute of Electromagnetic Engineering and Science (KIEES) and Dean of Information and Telecommunications College at Soongsil University. He is the director of the Wireless Power Transfer Research Center, supported by the Korean government's Ministry of Trade, Industry and Energy, and the director of the Metamaterials Research Center, supported by Basic Research Laboratories (BRL) through an NRF grant funded by the MSIP. He is the director of the Center for Intelligent Biomedical Wireless Power Transfer supported by the National Research Foundation of Korea (NRF) grant funded by the MSIP. His research interests include wireless technologies, RF power amplifiers, and wireless power transfer using metamaterials.

\section{Daesik Jeong}

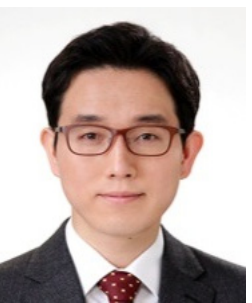

received the B.S. degree in electric wave engineering from Korea Maritime and Ocean University, Pusan, Korea, in 2005, and the M.S. degree in electronic engineering from Kyungpook National University, Daegu, Korea, in 2007. He was worked Hanwha Systems. His research interests include phased array antenna design and radar system. 